\title{
Prognostic value of Rho GDP dissociation inhibitors in patients with hepatocellular carcinoma following liver transplantation
}

\author{
MING-CHUN LAI ${ }^{1}$, QIAN-QIAN ZHU ${ }^{2}$, KWABENA-GYABAAH OWUSU-ANSAH ${ }^{1}$, YANG-BO ZHU ${ }^{1}$, \\ ZHE YANG $^{1}$, HAI-YANG XIE ${ }^{1}$, LIN ZHOU ${ }^{1}$, LI-MING WU ${ }^{1}$ and SHU-SEN ZHENG ${ }^{1}$ \\ ${ }^{1}$ Division of Hepatobiliary and Pancreatic Surgery, Key Laboratory of Combined Multi-organ Transplantation, \\ Ministry of Public Health, Department of Surgery; ${ }^{2}$ Department of Urinary Surgery, The First Affiliated Hospital, \\ Zhejiang University School of Medicine, Hangzhou, Zhejiang 310003, P.R. China
}

Received October 15, 2015; Accepted April 4, 2017

DOI: $10.3892 / \mathrm{ol} .2017 .6333$

\begin{abstract}
Rho GDP dissociation inhibitors (GDIs) are pivotal regulators of Rho GTPases, which are essential for tumor progression, yet their role in hepatocellular carcinoma (HCC) remains poorly understood. The purpose of the present study was to assess the role of RhoGDIs in the invasiveness and migration of liver cancer, and to determine their clinical prognostic significances in HCC following liver transplantation (LT). In the present study, the expression of RhoGDIs was assessed using reverse transcription-quantitative polymerase chain reaction and confirmed by western-blot analysis and immunohistochemistry. Their prognostic values were also analyzed, and determined in patients treated with LT. In addition, the functions of RhoGDIs in liver cancer cell line were studied in vitro. As a result, the downregulation of RhoGDI1 and RhoGDI2 at mRNA and protein levels were detected in HCC when compared with that of adjacent noncancerous tissues $(\mathrm{P}<0.05)$. However, the level of RhoGDI3 was identified to be similar in tumor and para-carcinoma tissues. Additionally, Kaplan-Meier curves demonstrated that patients with lower expression of RhoGDI1 or RhoGDI2 exhibited significantly increased risk of tumor recurrence following LT ( $\mathrm{P}=0.007$ and $\mathrm{P}=0.006$, respectively). Cox proportional hazards model analysis revealed that the decreased expression level of RhoGDI2 was an unfavorable independent prognostic factor (hazard ratio, $3.306 ; \mathrm{P}=0.001$ ). In vitro studies involving the silencing of RhoGDI1 or RhoGDI2 demonstrated a significant increase in the migratory and invasive ability of
\end{abstract}

Correspondence to: Dr Shu-Sen Zheng, Division of Hepatobiliary and Pancreatic Surgery, Key Laboratory of Combined Multi-organ Transplantation, Ministry of Public Health, Department of Surgery, The First Affiliated Hospital, Zhejiang University School of Medicine, 79 Qingchun Road, Shangcheng, Hangzhou, Zhejiang 310003, P.R. China

E-mail: shusenzheng@zju.edu.cn

Key words: Rho guanosine diphosphate dissociation inhibitors 1, RhoGDI2, hepatocellular carcinoma, liver transplantation, prognosis tumor cells upon the silencing of these genes. Results from the present study indicate that RhoGDI dysregulation is a frequent event in human $\mathrm{HCC}$, and that it promotes cancer progression by stimulating cell migration and invasion. RhoGDI 2 may be a prognostic biomarker for patients with HCC following LT, and act as a potential therapeutic target.

\section{Introduction}

Hepatocellular carcinoma (HCC) is currently the fifth most common type of solid tumor worldwide and the second leading cause of cancer-associated mortality in China, accounting for an estimated $>650,000$ mortalities annually (1). The high mortality associated with this disease may be attributed primarily to the inability to diagnose patients with HCC at an early stage, and its aggressive invasiveness and metastasis (2). Despite the fact that liver transplantation (LT) is considered the best treatment option for progressive HCC in selected patients accompanied by cirrhosis and hepatitis, the high postoperative recurrence and metastasis of HCC, due to its invasion-associated spreading, remains the biggest obstacle that affects the long-term prognosis of patients following LT $(3,4)$. This unfavorable prognosis is primarily due to the fact that HCC is a highly vascularized tumor type with frequent intra- or extra-hepatic metastases. However, at present, the molecular mechanisms underlying $\mathrm{HCC}$ recurrence and metastasis remain to be elucidated. In addition, there is an urgent demand to identify additional diagnostic and prognostic biomarkers of $\mathrm{HCC}$, which may be critical for developing novel therapeutic strategies.

Rho family GTPases, including Ras homolog gene family, member A, Ras-related C3 botulinum toxin substrate 1 and cell division control protein 42 homolog (Cdc42), belong to the small GTPases of the Ras oncogene superfamily that are known to have an essential role in cellular function. Their biological activities are controlled through a tightly regulated GDP/GTP cycle, which is stimulated by guanine nucleotide exchange factors and terminated by GTPase-activating proteins (5-7). As the pivotal suppressor of Rho GTPase, Rho GDP dissociation inhibitors (GDIs) bind to the majority of Rho GTPases in the cytoplasm to prevent the nucleotide exchange, and thus block their activation, which leads to carcinogenesis and promotes the invasive phenotype of tumor cells (8-10). At present, three 
human RhoGDIs have been identified, termed RhoGDI1 (RhoGDI1 or RhoGDI- $\alpha$ ), RhoGDI2 (D4-GDI or RhoGDI- $\beta$ ) and RhoGDI3 (RhoGDIG or RhoGDI- $\gamma$ ) (11). Dysregulation of RhoGDIs has been explored in a variety of human tumors; however, the expression levels exhibit opposite patterns depending on the tumor types. RhoGDI1 is upregulated in advanced ovarian and colorectal cancers and is associated with tumor progression $(12,13)$. However, the downregulation of RhoGDI1 has been identified in breast cancer, prostate cancer and HCC (14-16). RhoGDI2 is downregulated and identified as a putative metastasis suppressor in bladder cancer, colorectal carcinoma and Hodgkin's lymphoma, while the overexpression of RhoGDI2 has also been detected in cases of ovarian, breast and gastric cancer (12,17-20). However, RhoGDI3, which is preferentially expressed in the brain and pancreas, is present at low levels in patients with breast cancer metastasis (21). In addition, a small number of studies are available on the expression pattern and clinical implications of RhoGDIs in patients with HCC, while the role of RhoGDIs in tumor progression and metastasis remains controversial (22-24).

The purpose of the present study was to assess the role RhoGDIs perform in the invasiveness and migration of HCC, to investigate the expression of RhoGDIs in patients with $\mathrm{HCC}$ and to determine their clinical prognostic significance in HCC following LT. It was identified that the downregulation of RhoGDIs was associated with decreased recurrence-free survival for patients with HCC following LT, and promoted cancer progression by facilitating cell migration and invasion, supporting its role as a metastasis suppressor and its potential clinical utility for biotherapy.

\section{Materials and methods}

Clinical samples. In total, 138 patients were enrolled in the present study. A total of 80 hepatocellular carcinoma tissues were obtained from patients treated with orthotopic LT (OLT) between January 2002 and December 2005 in a single group at the First Affiliated Hospital of Zhejiang University School of Medicine (Hangzhou, China), among which 56 patients $(70 \%)$ were beyond the Milan criteria (3) and 45 patients $(56.3 \%)$ developed tumor recurrence during a follow-up period of 2-46.9 months. The median follow-up time was 19.2 months. All diagnosed cases were verified histologically prior or subsequent to surgery, and the clinicopathological variables were summarized in Table I. All patients were monitored by serum $\alpha$ fetoprotein (AFP) levels, abdominal ultrasonography, chest X-ray and computed tomography scanning regularly following LT for the surveillance of recurrence or metastases. Tumor recurrence was confirmed by radiological techniques and aided by serum AFP examination. An additional 58 matched pairs of HCC samples and adjacent liver tissues were also included in the present study. Tissues were immediately frozen in liquid nitrogen following surgery, and then stored at $-80^{\circ} \mathrm{C}$ until processing. The present study was approved by the Ethics Committee of Zhejiang University and written informed consent was obtained from all the patients, according to the Declaration of Helsinki.

Reverse transcription-quantitative polymerase chain reaction (RT-qPCR). Total RNA was extracted from all specimens using TRIzol reagent (Invitrogen; Thermo Fisher Scientific, Inc., Waltham, MA, USA) while RT-PCR was performed using the MMLV Reverse Transcriptase cDNA kit (Promega Corporation, Madison, WI, USA), according to the manufacturer's protocol. RT-qPCR Amplification was initiated with a 3-min pre-denaturation step at $93^{\circ} \mathrm{C}$, followed by 40 cycles of $1 \mathrm{~min}$ at $93^{\circ} \mathrm{C}, 1 \mathrm{~min}$ at $55^{\circ} \mathrm{C}$, and $1 \mathrm{~min}$ at $72^{\circ} \mathrm{C}$ and then a final extension at $72^{\circ} \mathrm{C}$ for $5 \mathrm{~min}$. SYBR-Green (Applied Biosystems; Thermo Fisher Scientific, Inc.) was used as a fluorophore. qPCR was performed in triplicate using an ABI7500 system (Applied Biosystems; Thermo Fisher Scientific, Inc.). The expression of RhoGDIs was normalized to $\beta$-actin, which was used as an internal control according to the $2^{-\Delta \Delta \mathrm{Cq}}$ method (25). All primer sequences used in this study were as follows: RhoGDI1 sense, 5'-ACTACAAGCCCCCGGCCCAG-3' and antisense, 5'-GTT GGGGACGTTGGGGTCTGC-3'; RhoGDI2 sense, 5'-GAG AGACAGAGGCACCCCGGA-3' and antisense, 5'-GCTTTC GGATCTGTCACCACAGGA-3'; RhoGDI3 sense, 5'-TGT CGGAACAGGCTCCGGGG-3' and antisense, 5'-GACGGT CTTGTCCACGCGCA-3'; and $\beta$-actin sense, 5'-TTGTTA CAGGAAGTCCCTTGCC-3' and antisense, 5'-ATGCTATCA CCTCCCCTGTGTG-3' (26). All reactions were performed in duplicate.

Western blot analysis. Clinically frozen tissues were homogenized in radioimmunoprecipitation lysis buffer and incubated at $4^{\circ} \mathrm{C}$ for $3 \mathrm{~h}$. For electrophoresis, total protein ( $40 \mu \mathrm{g}$ per lane) was separated by $12 \%$ SDS-PAGE (Invitrogen; Thermo Fisher Scientific, Inc.) and then transferred onto polyvinylidene fluoride membranes. Following blotting, the membranes were incubated with rabbit monoclonal antibody against human RhoGDI1 (1:1,000, cat. no. ab133248; Abcam, Cambridge, UK), rabbit polyclonal antibody against RhoGDI2 (1:2,000, cat. no. ab15198; Abcam) or mouse monoclonal antibody against $\beta$-actin (1:2,000, cat. no. sc130065; Santa Cruz Biotechnology, Inc., Dallas, TX, USA) overnight at $4^{\circ} \mathrm{C}$. Following 3 washes with TBST buffer $(20 \mathrm{mM}$ Tris- $\mathrm{HCl}, 150 \mathrm{mM} \mathrm{NaCl}$, and $0.05 \%$ Tween-20) for $10 \mathrm{~min}$, the membranes were incubated with second antibody for $1 \mathrm{~h}$ at room temperature. Finally, immunocomplexes were visualized by chemiluminescence using enhanced chemiluminescence (Applygen Technologies, Inc., Beijing, China) according to the manufacturer's protocol.

Immunohistochemical analysis. Immunohistochemical studies on RhoGDIs were performed on formalin-fixed tissue sections obtained from tumor tissues and their paired adjacent non-tumors tissues. In brief, $4 \mu \mathrm{m}$ sections were heated at $60^{\circ} \mathrm{C}$ for $20 \mathrm{~min}$ followed by deparaffinization with xylene for $20 \mathrm{~min}$ and rehydration with a graded ethanol series $(100,95$, 80 and $70 \%$ ) and deionized distilled water, for 5 min each. Subsequent to treatment with $3 \%$ hydrogen peroxide to quench endogenous peroxidase activity, the sections were submerged into potassium citrate antigenic retrieval buffer and microwaved for antigenic retrieval, after which they were incubated with $1 \%$ bovine serum albumin (Gibco; Thermo Fisher Scientific, Inc.) to block non-specific binding sites. Then, the sections were incubated with primary antibodies against RhoGDI1 (1:50; cat. no. ab133248; Abcam) or RhoGDI2 (1:100; cat. no. ab15198; Abcam) for $1 \mathrm{~h}$ at $37^{\circ} \mathrm{C}$. Following 3 washes with phosphate buffered saline (PBS) for $5 \mathrm{~min}$, tissue slides 
Table I. Association between RhoGDIs and clinicopathological variables of patients with hepatocellular carcinoma following liver transplantation.

\begin{tabular}{|c|c|c|c|c|c|c|c|c|c|}
\hline \multirow[b]{2}{*}{ Variables } & \multicolumn{2}{|c|}{$\begin{array}{c}\text { RhoGDI1 } \\
\text { expression, } \mathrm{n}\end{array}$} & \multirow[b]{2}{*}{ P-value ${ }^{a}$} & \multicolumn{2}{|c|}{$\begin{array}{c}\text { RhoGDI2 } \\
\text { expression, } \mathrm{n}\end{array}$} & \multirow[b]{2}{*}{ P-value } & \multicolumn{2}{|c|}{$\begin{array}{c}\text { RhoGDI3 } \\
\text { expression, } \mathrm{n}\end{array}$} & \multirow[b]{2}{*}{$P$-value } \\
\hline & Low & High & & Low & High & & Low & High & \\
\hline \multicolumn{10}{|l|}{ Age } \\
\hline$\leq 50$ years & 24 & 21 & 0.795 & 27 & 19 & 0.062 & 19 & 21 & 0.118 \\
\hline$>50$ years & 16 & 19 & & 13 & 21 & & 21 & 13 & \\
\hline \multicolumn{10}{|l|}{ Sex } \\
\hline Female & 3 & 4 & $>0.999$ & 4 & 3 & $>0.999$ & 3 & 4 & $>0.999$ \\
\hline Male & 37 & 36 & & 36 & 37 & & 37 & 36 & \\
\hline \multicolumn{10}{|l|}{ PVTT } \\
\hline Absent & 25 & 28 & 0.785 & 27 & 27 & $>0.999$ & 29 & 24 & 0.273 \\
\hline Present & 15 & 12 & & 13 & 13 & & 11 & 16 & \\
\hline \multicolumn{10}{|c|}{ Preoperative AFP level } \\
\hline$\leq 400 \mathrm{ng} / \mathrm{ml}$ & 20 & 24 & 0.604 & 23 & 21 & 0.795 & 21 & 23 & 0.795 \\
\hline$>400 \mathrm{ng} / \mathrm{ml}$ & 20 & 16 & & 17 & 19 & & 19 & 17 & \\
\hline \multicolumn{10}{|c|}{ Histopathological grading } \\
\hline Well + moderate & 27 & 29 & 0.779 & 24 & 32 & 0.091 & 32 & 24 & 0.128 \\
\hline Poor & 13 & 11 & & 16 & 8 & & 8 & 16 & \\
\hline \multicolumn{10}{|l|}{ Tumor size } \\
\hline$\leq 5 \mathrm{~cm}$ & 16 & 19 & 0.795 & 19 & 16 & 0.795 & 12 & 21 & 0.063 \\
\hline$>5 \mathrm{~cm}$ & 24 & 21 & & 21 & 24 & & 28 & 19 & \\
\hline \multicolumn{10}{|l|}{ Tumor number } \\
\hline Single & 13 & 13 & $>0.999$ & 13 & 13 & $>0.999$ & 16 & 11 & 0.273 \\
\hline Multiple & 27 & 27 & & 27 & 27 & & 24 & 19 & \\
\hline \multicolumn{10}{|l|}{ Tumor recurrence } \\
\hline No & 9 & 24 & $0.004^{\mathrm{a}}$ & 11 & 23 & $0.018^{\mathrm{a}}$ & 20 & 13 & 0.190 \\
\hline Yes & 31 & 16 & & 29 & 17 & & 20 & 27 & \\
\hline
\end{tabular}

${ }^{a}$ The data were analyzed by $\chi^{2}$ test or Fisher exact test. RhoGDI, Rho GDP dissociation inhibitor; PVTT, portal vein tumor thrombosis; AFP, $\alpha$ fetoprotein.

were treated with biotinylated secondary anti-rabbit antibody (1:200; cat. no. a11034; Invitrogen; Thermo Fisher Scientific, Inc.) and horseradish peroxidase (HRP)-streptavidin complex (Yeasen, Shanghai, China) at $37^{\circ} \mathrm{C}$ for $30 \mathrm{~min}$ each. Finally, all sections were counterstained with hematoxylin, dehydrated, and mounted. The immunostained scores were independently interpreted and categorized using a semi-quantitative scale as follows: $0, \leq 5 \%$ positive staining; $1,6-25 \%$ positive; $2,26-50 \%$ positive; $3,51-75 \%$ positive; and $4,>75 \%$ positive by two pathologists blinded to the clinical data.

Cell culture. Human liver cancer HepG2 and MHCC-97L cell lines were purchased from the American Type Culture Collection (Manassas, VA, USA) and Liver Cancer Institute of Fudan University (Shanghai, China), respectively. All the cell lines were maintained in Dulbecco's modified Eagle's Medium (DMEM) (Gibco; Thermo Fisher Scientific, Inc.) with high glucose, supplemented with $10 \%$ fetal bovine serum (FBS) (Gibco; Thermo Fisher Scientific, Inc.) at $37^{\circ} \mathrm{C}$ in a $5 \% \mathrm{CO}_{2}$ atmosphere at constant humidity.
RNA interference experiments. All small interfering RNAs (siRNAs) were purchased from Shanghai GenePharma Co., Ltd. (Shanghai, China). Transient transfection of each siRNA to knock down RhoGDIs using Lipofectamine 2000 transfection reagent (Invitrogen; Thermo Fisher Scientific, Inc.), according to the manufacturer's protocol. The siRNA sequences were as follows: RhoGDI1-siRNA, 5'-AATTTA AGCAGTTAGAACT-3'; RhoGDI2-siRNA1, 5'-AATACG TTCAGCACACCTACA-3'; RhoGDI2-siRNA2, 5'-AAG GAAGGTTCTGAATATAGA-3' and scrambled negative control-siRNA, 5'-AATCGCATAGCGTATGCCGTT-3'. The cells were harvested following $48 \mathrm{~h}$ of transfection, and the efficiency of each siRNA oligo duplex was confirmed by qPCR.

In vitro cell migration/invasion assay. Cell migration and invasion were measured by an in vitro Transwell assay (Merck KGaA, Darmstadt, Germany), according to the manufacturer's protocol. Tumor cells $\left(2.5 \times 10^{5}\right)$ transfected with RhoGDI1/2-siRNA were suspended in $250 \mu \mathrm{l}$ serum-free DMEM were placed in 

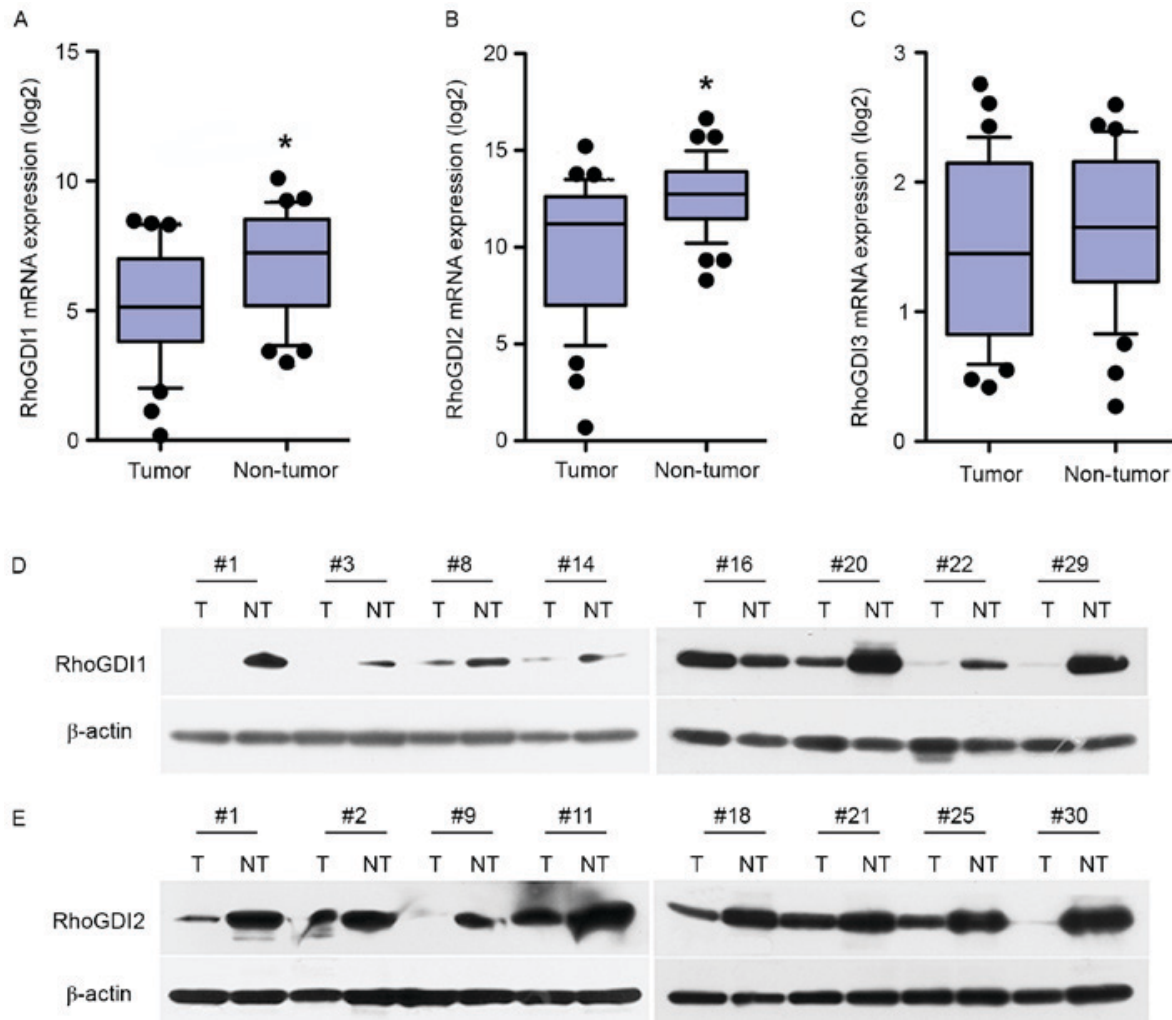

Figure 1. Expression of RhoGDIs in HCC and precancerous tissues. (A) Significantly lower expression of RhoGDI1 and (B) RhoGDI2 mRNA was detected in tumors than in paired non-tumor tissue. (C) There was no significant difference in RhoGDI3 mRNA expression between tumor and non-tumor groups. (D) Protein levels of RhoGDI1 and (E) RhoGDI2 were downregulated in tumors compared with non-tumor tissue, as detected by western blot analysis *P<0.05. RhoGDI, Rho GDP dissociation inhibitor; HCC, hepatocellular carcinoma; T, tumor; NT, non-tumor.

the upper insert, whose porous membrane was coated with (for invasion assay) or without (for migration assay) Matrigel (BD Biosciences, Franklin Lakes, NJ, USA). Subsequently, $600 \mu \mathrm{l}$ medium containing $10 \%$ FBS was added to the lower chamber as a chemoattractant. Following migration for $24 \mathrm{~h}$, or invasion for $48 \mathrm{~h}$, the insert was removed and added into dried methanol for $15 \mathrm{~min}$ at room temperature. Finally, the penetrated cells on the filters were stained in $0.1 \%$ crystal violet and counted in 5 fields under a x100 objective lens of an Olympus CX31 light microscope (Olympus Corporation, Tokyo, Japan). Tumor cells transfected with negative control-siRNA were used as control. Each experiment setting was repeated in triplicate.

Statistical analysis. All statistical data were processed with SPSS v.16.0 (SPSS, Inc., Chicago, IL) and GraphPad Prism v5.0 (GraphPad Software, La Jolla, CA) software. The differences between the two groups were analyzed using an unpaired Student's t-test. The $\chi^{2}$ or Fisher's exact tests were used for comparisons between RhoGDI expression and clinicopathological factors. Tumor-free survival curves were drawn by the Kaplan-Meier method, and compared by the log-rank test. Cox regression analysis was used for univariate and multivariate analysis to calculate the hazard ratios for risk factors. $\mathrm{P}<0.05$ was considered to indicate a statistically significant difference.

\section{Results}

Expression of RhoGDIs in HCC and precancerous tissues. For the analysis of RhoGDIs expression in HCC, the expression of
RhoGDIs was first examined in 58 pairs of HCC tissues and adjacent noncancerous liver tissues by qPCR. As expected, the downregulation of RhoGDI1 and RhoGDI2 mRNA was observed in $39(67.2 \%)$ and 38 patients $(65.5 \%)$, respectively. Overall, GDI1 and GDI2 expression was significantly decreased in tumors compared with matched non-tumorous tissues $(\mathrm{P}<0.05$; Fig. 1A and $\mathrm{B})$; however, RhoGDI3 expression did not differ significantly between tumor and non-tumor tissue ( $\mathrm{P}>0.05$; Fig. 1C). These results were then confirmed in 30 pairs of primary HCC tissues by western blot analysis. Compared with paired non-tumor liver tissues, the downregulation of RhoGDI1 and RhoGDI2 were observed in 20/30 (66.7\%) and 21/30 (70\%) of HCCs, respectively (Fig. 1D and E). In addition, the expression and subcellular localization of RhoGDIs protein was determined by immunohistochemistry, and its expression intensity corresponded closely with those of the RT-qPCR and immunoblotting analysis (Fig. 2).

Association of RhoGDI expression with clinicopathological variables. To determine the clinical significance of RhoGDIs in HCC, the expression of RhoGDIs in 80 consecutive patients who underwent LT was examined. When patients were segregated into low/high expression groups with the median GDI expression level as the cut-off line, the association study demonstrated that a low expression of RhoGDI1 and RhoGDI2, but not RhoGDI3, was significantly associated with tumor recurrence following LT (Table I). However, no significant association was observed between the downregulation of RhoGDIs and clinicopathological characteristics, 


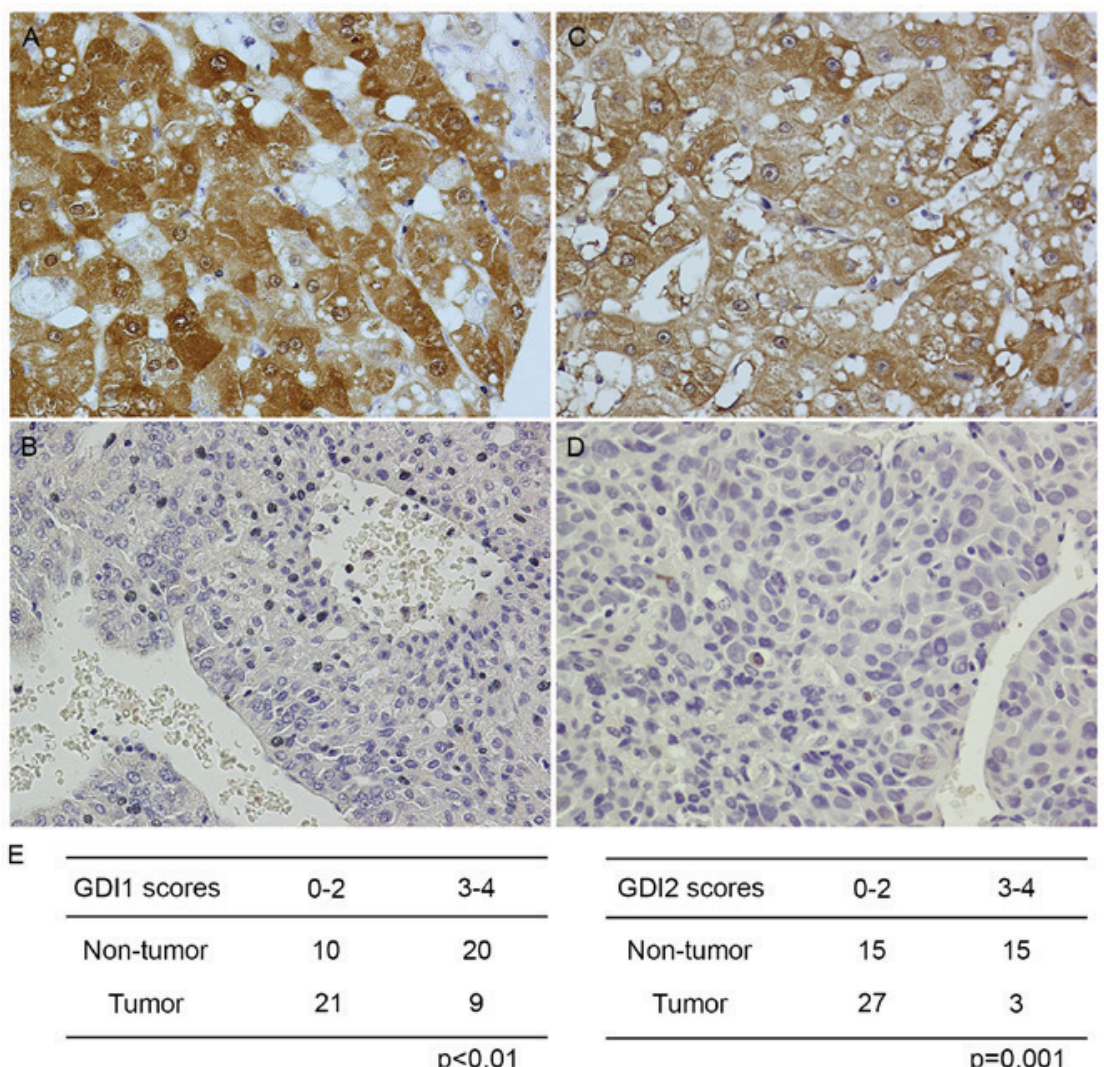

Figure 2. Expression of RhoGDI1 and RhoGDI2 was downregulated in HCC. (A) Expression of RhoGDI1 in para-carcinoma tissues and (B) HCC. (C) Expression of RhoGDI2 in para-carcinoma tissues and (D) HCC. (E) RhoGDI1 and RhoGDI2 protein expression scores were significantly decreased in tumors compared with matched non-tumorous tissues. RhoGDI, Rho GDP dissociation inhibitor; HCC, hepatocellular carcinoma.

which included age, gender, AFP level, histopathological grading, tumor number, tumor size and portal vein tumor thrombus (Table I).

RhoGDI expression is associated with HCC prognosis. Whether the downregulation of RhoGDIs was associated with the prognosis of patients with HCC was investigated. Notably, Kaplan-Meier survival curves and the log-rank tests indicated that the low expression of RhoGDI1 and RhoGDI2 were unfavorable prognostic predictors for the disease-free survival (DFS) of HCC, whereas RhoGDI3 expression exhibited no significant association with HCC prognosis (RhoGDI1, $\mathrm{P}=0.02$; RhoGDI2, $\mathrm{P}=0.006$; RhoGDI3, $\mathrm{P}=0.108$; Fig. 3A-C). The combined effect of RhoGDI1 and RhoGDI2 mRNA on DFS of HCC was also evaluated. The GDI1 and GDI2 low expression group demonstrated significantly shorter DFS compared with other groups (either GDI1 or GDI2 high expression group; GDI1 and GDI2 high group; Fig. 3D). Concurrently, univariate analyses were performed to determine the prognostic factors for predicting tumor recurrence of HCC by the Kaplan-Meier method. As demonstrated in Table II, three of the well-known factors associated with $\mathrm{HCC}$ recurrence were confirmed in the present study (tumor size; preoperative AFP level; portal vein tumor thrombus). In addition, the downregulation of RhoGDI1 or RhoGDI2 exhibited significantly poorer DFS compared with those patients with high expression of RhoGDI1 or RhoGDI2 (Table II). Ultimately, by the multivariate Cox regression analysis, apart from preoperative serum AFP level, low levels of expression RhoGDI2 were identified to be an independent predictor of recurrence-free survival for HCC following LT (hazard ratio, 3.306; 95\% confidence interval, 1.610-6.790; P=0.001; Table II). The data of the present study indicate that RhoGDI2 expression may be an important indicator for predicting HCC recurrence, which in turn may affect the overall survival of patients following LT.

Effects of RhoGDIs on cell migration and invasion. It has been demonstrated that RhoGDI1 and RhoGDI2 function as metastasis suppressors and may block cell invasion in different types of tumors. To explore whether RhoGDI depletion affects cell migration and invasion, a Transwell assay was performed in GDI-RNAi cells. Following $48 \mathrm{~h}$ of RNA interference, expression of RhoGDI1 and RhoGDI2 was efficiently suppressed by specific siRNAs. As demonstrated in Fig. 4, HepG2 cells with RhoGDI1 knockout exhibited significantly higher rates of migration and invasion compared with control groups. Coincidently, silencing RhoGDI2 expression effectively enhanced Matrigel invasion activity of HepG2 cells $(\mathrm{P}<0.05$; Fig. 4A and B). Similar results were observed in MHCC-97L cells (data not shown). Overall, these in vitro studies demonstrated that RhoGDIs perform crucial roles in the regulation of HCC cell motility and invasiveness.

\section{Discussion}

Although marked progress has been made in previous decades, the survival of patients with $\mathrm{HCC}$ remains at $~ 50 \%$ (range, 17-69) following 5 years $(27,28)$. Tumor recurrence remains 

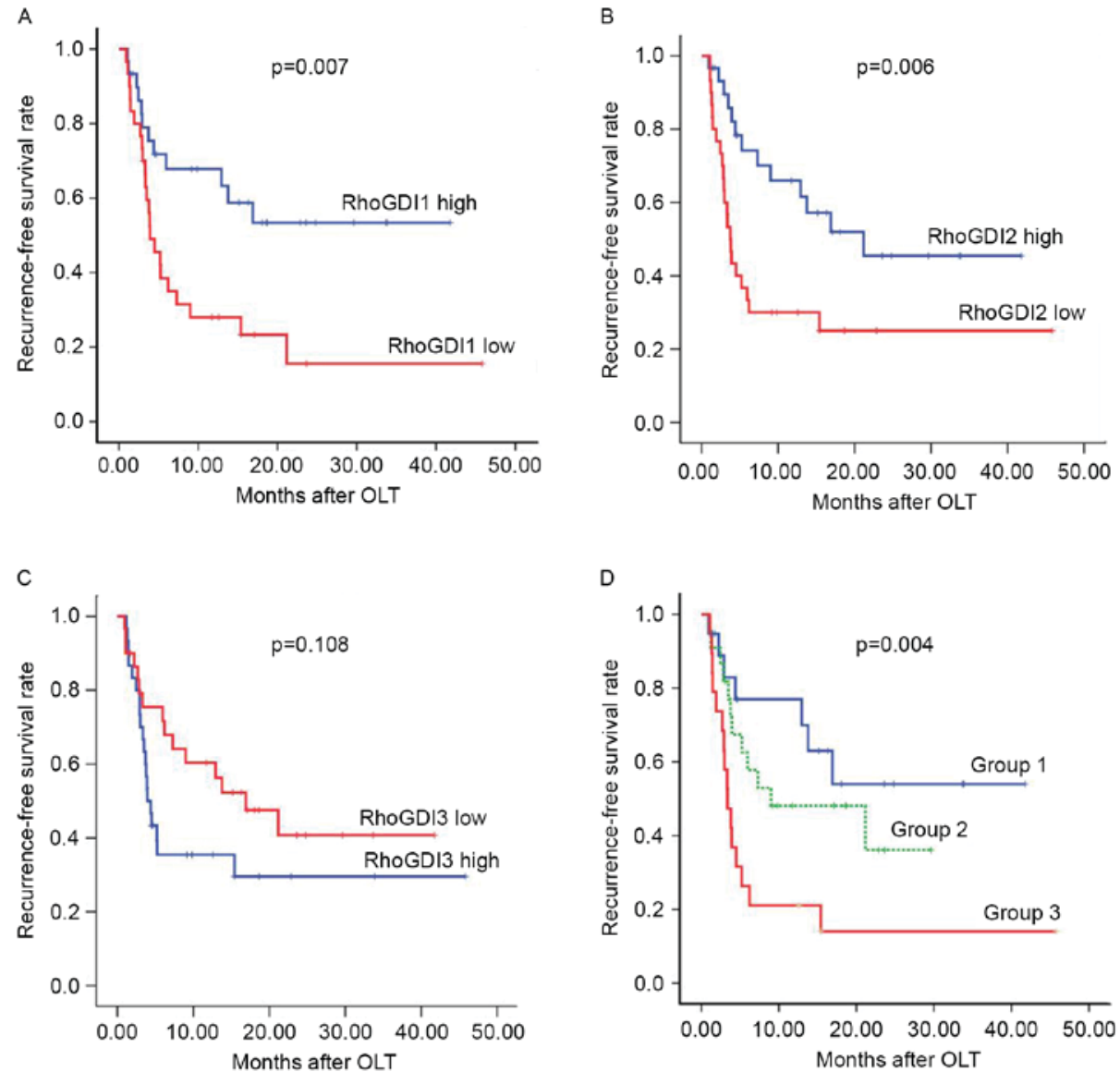

Figure 3. Kaplan-Meier analysis of recurrence-free survival rates in HCC patients following OLT. (A) Recurrence-free survival time of patients according to the expression levels of RhoGDI1, (B) RhoGDI2 and (C) RhoGDI3. (D) The recurrence-free survival rates were significantly lower in GDI1 and GDI2 low-expression group compared with other groups (either GDI1 or GDI2 high-expression group, or the GDI1 and GDI2 high-expression group). RhoGDI, Rho GDP dissociation inhibitor; HCC, hepatocellular carcinoma; OLT, orthotopic liver transplantation.

one of the major challenges for patients with HCC undergoing LT, constituting a major factor in its poor prognosis. Diverse significant risk factors for recurrence have been established, including tumor rupture, venous invasion and cirrhosis, which have been implicated in early recurrence (29). Viral replication in viral hepatitis and multiple tumors increases the risk for the late recurrence $(30,31)$. However, the molecular pathogenesis underlying tumor recurrence remains largely unknown, which seriously hinders the improvement of the clinical management of HCC $(32,33)$. In previous years, it was widely accepted that abnormal gene expression constituted one of the major characteristics of tumor invasion and metastasis. Thus, to elucidate the mechanisms and identify novel biomarkers underlying the early recurrence of $\mathrm{HCC}$ are considered to be significant for improvements to the therapeutic interventions and overall prognosis for patients with HCC.

Rho GTPases, including Rho, Rac and $\mathrm{Cdc} 42$, that bind RhoGDIs regulate numerous cellular functions, including cell polarity, cell cycle progression, apoptosis, vesicular trafficking and tumorigenesis in several types of cancer $(5,13,16)$. Changes in the levels of the different RhoGDIs markedly affects the overall levels and Rho GTPase activity, primarily by their ability to prevent nucleotide exchange and membrane association (9). This indicates that the deregulation of RhoGDIs may be involved in progression and metastasis for a number of types of human cancer.
To the best of our knowledge, the role of RhoGDIs in cancer remains controversial. A small number of studies mentioned the expression of RhoGDIs in HCC. Ding et al (22) identified that miR-151 may directly target RhoGDI1, which is frequently downregulated in HCC and functions as a metastasis suppressor, leading to the activation of Rac1, Cdc42 and Rho GTPases, thus facilitating HCC cell invasion and spreading (22). In a small study using transcriptomic and proteomic approaches, the downregulation of RhoGDI2 was identified in patients with HCC with hepatitis B infection (24). In the present study, the expression of RhoGDI1 and RhoGDI2, but not RhoGDI3, was significantly lower in HCC tissues compared with para-cancerous tissues at mRNA and protein levels, and these results are consistent with a previous study (16). In addition, a lower expression of RhoGDI1 was negatively associated with disease-free survival in patients with HCC who underwent LT $(\mathrm{P}=0.013)$. Since Rho-GDI2 and Rho-GDI1 exhibit similar abilities to inhibit Rho GTPase, it was hypothesized whether Rho-GDI2 would exert the same effect on HCC prognosis. According to the present study, the same trend was also observed at the RhoGDI2 gene $(\mathrm{P}=0.006)$. The most valuable result of the present study is that RhoGDI2 expression is an independent prognostic factor for $\mathrm{HCC}$ recurrence following LT. To the best of our knowledge, this is the first study demonstrating that RhoGDI2 is frequently downregulated in $\mathrm{HCC}$, and functions as a metastasis suppressor in HCC. It also 
Table II. Downregulation of RhoGDI2 is an independent prognostic factor for patients with hepatocellular carcinoma following liver transplantation.

\begin{tabular}{lcc}
\hline Variables & Hazard ratio $(95 \%$ confidence interval $)$ & P-value $^{\mathrm{a}}$ \\
\hline Univariate analysis & - & - \\
Tumor size $(>5 \mathrm{~cm}$ vs. $\leq 5 \mathrm{~cm})$ & - & 0.034 \\
AFP $(>400 \mathrm{ng} / \mathrm{ml}$ vs. $\leq 400 \mathrm{ng} / \mathrm{ml})$ & - & 0.006 \\
PVTT (present vs. absent) & - & 0.028 \\
RhoGDI1 expression (low vs. high) & - & 0.007 \\
RhoGDI2 expression (low vs. high) & - & - \\
Multivariate analysis & $2.482(1.207-5.012)$ & 0.006 \\
AFP $(>400 \mathrm{ng} / \mathrm{ml}$ vs. $\leq 400 \mathrm{ng} / \mathrm{ml})$ & $3.306(1.610-6.790)$ & 0.001 \\
RhoGDI2 expression (low vs. high) & -13 \\
\hline
\end{tabular}

${ }^{a}$ Log-rank test was used for univariate analysis and Cox hazard ratio was used for multivariate analysis. RhoGDI, Rho GDP dissociation inhibitor; PVTT, portal vein tumor thrombosis; AFP, $\alpha$ fetoprotein.

A

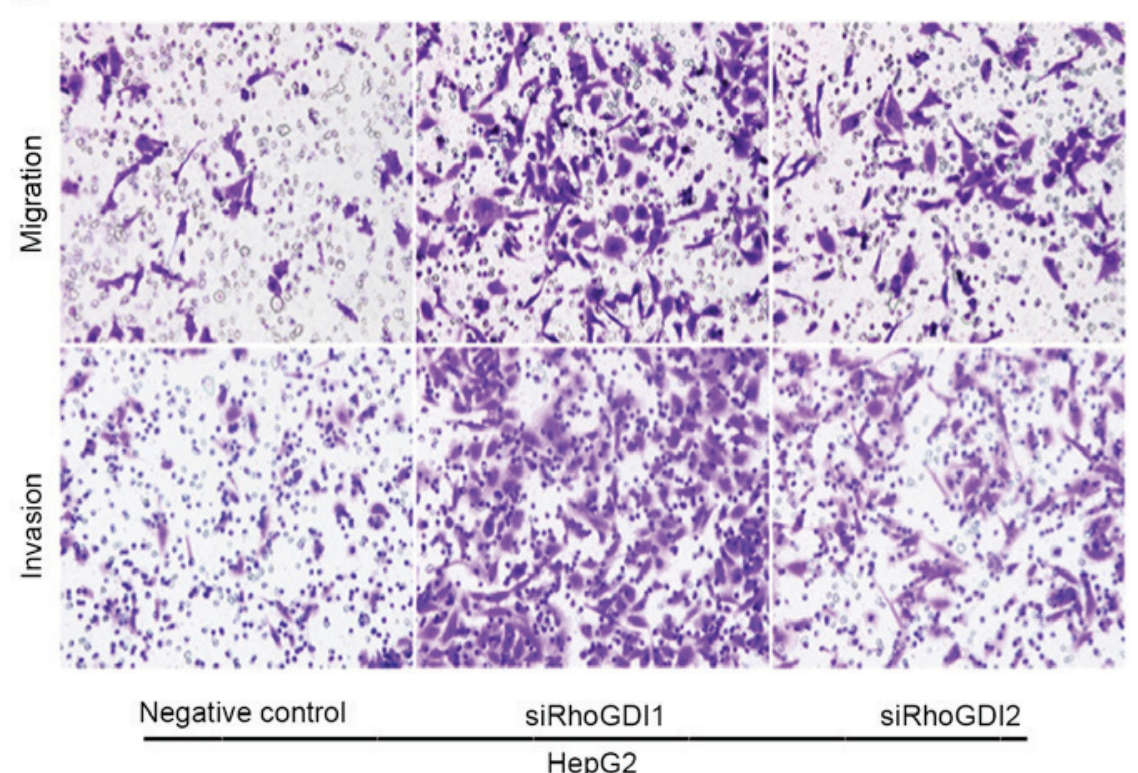

B
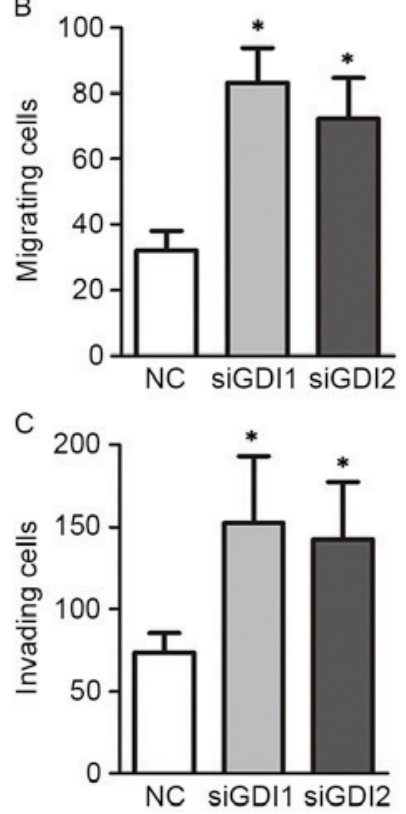

Figure 4. Assessment of biological roles of RhoGDI1 and RhoGDI2 by in vitro assays in HepG2 cells. (A) Silencing RhoGDI1 or RhoGDI2 expression effectively enhanced migration and Matrigel invasion activity of HepG2 cells. (B) Quantitative scale of migration and (C) Matrigel invasion activity, which significantly increased in tumor cells treated with siRhoGDI. "P<0.05. RhoGDI, Rho GDP dissociation inhibitor; siRhoGDI1, small interfering RNA targeting RhoGDI1.

possesses negative associations with recurrence-free survival of patients with HCC following LT. Thus, detecting the expression levels of RhoGDI1, in combination with RhoGDI2 and Rho GTPases, may be valuable in predicting the prognosis of patients with HCC prior to LT more accurately compared with current measures. Future studies will determine whether the deregulation of Rho GTPase activity is also associated with the prognosis of patients with HCC following LT. By contrast, Rho-GDI3 did not affect the clinical outcome of HCC, implying that besides sharing functions, the three RhoGDIs may also have individual, not interchangeable, activities in tumor progression.

Based on the trend of decreasing RhoGDI expression in $\mathrm{HCC}$ and poorer clinical prognosis, it was considered that lower expression levels of RhoGDI in tumor cells may contribute to more aggressive tumor behavior. To confirm this hypothesis, the biological significance of RhoGDI in cancer invasion and migration was then investigated in vitro. It was observed that the knockdown of RhoGDI1 and RhoGDI2 by siRNA accelerated the cell migration rate, and the invasive ability of HepG2 and MHCC-97L cells. Despite the critical molecular mechanism underlying the biological function of GDIs, which has not been described in the present study, to a certain extent it indicates that the loss of RhoGDIs promotes the malignant behavior of HCC cells. In the future, additional molecular and animal model studies are also necessary to confirm these data.

In summary, the results of the present study demonstrate the important roles RhoGDIs perform, particularly RhoGDI2, 
in liver cancer biology. The downregulation of RhoGDI1 and RhoGDI2 may significantly promote HCC cell invasion and migration in vitro. Concurrently, RhoGDI 2 may be a novel biomarker and independent prognostic factor in predicting the recurrence of HCC following LT. These data may enable an exploration of the potential use of RhoGDIs as a target for cancer therapy.

\section{Acknowledgements}

The present study was supported by grants from the National S\&T Major Project (no. 2012ZX10002017) and the National Natural Science Foundation of China (nos. 81101840 and 81302074). The abstract of the present study was presented at the International Liver Transplantation Society 20th Annual International Congress (June 2014; London, United Kingdom).

\section{References}

1. El-Serag HB and Rudolph KL: Hepatocellular carcinoma: Epidemiology and molecular carcinogenesis. Gastroenterology 132: 2557-2576, 2007.

2. Hainaut $P$ and Plymoth A: Targeting the hallmarks of cancer: Towards a rational approach to next-generation cancer therapy. Curr Opin Oncol 25: 50-51, 2013.

3. Mazzaferro V, Regalia E, Doci R, Andreola S, Pulvirenti A, Bozzetti F, Montalto F, Ammatuna M, Morabito A and Gennari L: Liver transplantation for the treatment of small hepatocellular carcinomas in patients with cirrhosis. N Engl J Med 334: 693-699, 1996.

4. Zheng SS, Xu X, Wu J, Chen J, Wang WL, Zhang M, Liang TB and $\mathrm{Wu} \mathrm{LM}$ : Liver transplantation for hepatocellular carcinoma: Hangzhou experiences. Transplantation 85: 1726-1732, 2008.

5. Liu J, Zhang D, Luo W, Yu Y, Yu J, Li J, Zhang X, Zhang B, Chen J, Wu XR, et al: X-linked inhibitor of apoptosis protein (XIAP) mediates cancer cell motility via Rho GDP dissociation inhibitor (RhoGDI)-dependent regulation of the cytoskeleton. J Biol Chem 286: 15630-15640, 2011.

6. Olofsson B: Rho guanine dissociation inhibitors: Pivotal molecules in cellular signalling. Cell Signal 11: 545-554, 1999.

7. Moissoglu K, McRoberts KS, Meier JA, Theodorescu D and Schwartz MA: Rho GDP dissociation inhibitor 2 suppresses metastasis via unconventional regulation of RhoGTPases. Cancer Res 69: 2838-2844, 2009.

8. Garcia-Mata R, Boulter E and Burridge K: The 'invisible hand': Regulation of RHO GTPases by RHOGDIs. Nat Rev Mol Cell Biol 12: 493-504, 2011.

9. DerMardirossian C and Bokoch GM: GDIs: Central regulatory molecules in Rho GTPase activation. Trends Cell Biol 15: 356-363, 2005.

10. Fang KP, Zhang JL, Ren YH and Qian YB: Talin-1 correlates with reduced invasion and migration in human hepatocellular carcinoma cells. Asian Pac J Cancer Prev 15: 2655-2661, 2014.

11. Lu W, Wang $\mathrm{J}$ and Wen T: Downregulation of Rho-GDI gamma promotes differentiation of neural stem cells. Mol Cell Biochem 311: 233-240, 2008.

12. Zhen H, Yang S, Wu H, Wang S, Lv J, Ma L and Zhang X: LyGDI is a promising biomarker for ovarian cancer. Int $\mathbf{J}$ Gynecol Cancer 20: 316-322, 2010.

13. Zhao L, Wang H, Sun X and Ding Y: Comparative proteomic analysis identifies proteins associated with the development and progression of colorectal carcinoma. FEBS J 277: 4195-4204, 2010.

14. Ronneburg H, Span PN, Kantelhardt E, Dittmer A, Schunke D, Holzhausen HJ, Sweep FC and Dittmer J: Rho GDP dissociation inhibitor $\alpha$ expression correlates with the outcome of CMF treatment in invasive ductal breast cancer. Int J Oncol 36: 379-386, 2010.
15. Yamashita T, Okamura T, Nagano K, Imai S, Abe Y, Nabeshi H, Yoshikawa T, Yoshioka Y, Kamada H, Tsutsumi Y and Tsunoda S: Rho GDP-dissociation inhibitor alpha is associated with cancer metastasis in colon and prostate cancer. Pharmazie 67: 253-255, 2012.

16. Li W, Wang H, Jin X and Zhao L: Loss of RhoGDI is a novel independent prognostic factor in hepatocellular carcinoma. Int $\mathrm{J}$ Clin Exp Pathol 6: 2535-2541, 2013.

17. Wu Y, McRoberts K, Berr SS, Frierson HF Jr, Conaway M and Theodorescu D: Neuromedin U is regulated by the metastasis suppressor RhoGDI2 and is a novel promoter of tumor formation, lung metastasis and cancer cachexia. Oncogene 26: 765-773, 2007.

18. Ma L, Xu G, Sotnikova A, Szczepanowski M, Giefing M, Krause K, Krams M, Siebert R, Jin J and Klapper W: Loss of expression of LyGDI (ARHGDIB), a rho GDP-dissociation inhibitor, in Hodgkin lymphoma. Br J Haematol 139: 217-223, 2007.

19. Moon HG, Jeong SH, Ju YT, Jeong CY, Lee JS, Lee YJ, Hong SC, Choi SK, Ha WS, Park ST and Jung EJ: Up-regulation of RhoGDI2 in human breast cancer and its prognostic implications. Cancer Res Treat 42: 151-156, 2010.

20. Cho HJ, Baek KE, Park SM, Kim IK, Choi YL, Cho HJ, Nam IK, Hwang EM, Park JY, Han JY, et al: RhoGDI2 expression is associated with tumor growth and malignant progression of gastric cancer. Clin Cancer Res 15: 2612-2619, 2009.

21. Zhang Y and Zhang B: D4-GDI, a Rho GTPase regulator, promotes breast cancer cell invasiveness. Cancer Res 66: 5592-5598, 2006

22. Ding J, Huang S, Wu S, Zhao Y, Liang L, Yan M, Ge C, Yao J, Chen T, Wan D, et al: Gain of miR-151 on chromosome 8q24.3 facilitates tumour cell migration and spreading through downregulating RhoGDIA. Nat Cell Biol 12: 390-399, 2010.

23. Wang H, Wang B, Liao Q, An H, Li W, Jin X, Cui S and Zhao L: Overexpression of RhoGDI, a novel predictor of distant metastasis, promotes cell proliferation and migration in hepatocellular carcinoma. FEBS Lett 588: 503-508, 2014.

24. Li C, Tan YX, Zhou H, Ding SJ, Li SJ, Ma DJ, Man XB, Hong Y, Zhang L, Li L, et al: Proteomic analysis of hepatitis B virus-associated hepatocellular carcinoma: Identification of potential tumor markers. Proteomics 5: 1125-1139, 2005.

25. Livak KJ and Schmittgen TD: Analysis of relative gene expression data using real-time quantitative PCR and the 2(-Delta Delta C(T)) Method. Methods 25: 402-408, 2001

26. Lai MC, Yang Z, Zhou L, Zhu QQ, Xie HY, Zhang F, Wu LM, Chen LM and Zheng SS: Long non-coding RNA MALAT-1 overexpression predicts tumor recurrence of hepatocellular carcinoma after liver transplantation. Med Oncol 29: 1810-1816, 2012.

27. Hanahan D and Weinberg RA: Hallmarks of cancer: The next generation. Cell 144: 646-674, 2011.

28. Yuan SX, Yang F, Yang Y, Tao QF, Zhang J, Huang G, Yang Y, Wang RY, Yang S, Huo XS, et al: Long noncoding RNA associated with microvascular invasion in hepatocellular carcinoma promotes angiogenesis and serves as a predictor for hepatocellular carcinoma patients' poor recurrence-free survival after hepatectomy. Hepatology 56: 2231-2241, 2012.

29. Livraghi T, Makisalo H and Line PD: Treatment options in hepatocellular carcinoma today. Scand J Surg 100: 22-29, 2011.

30. Fernandez M, Semela D, Bruix J, Colle I, Pinzani M and Bosch J: Angiogenesis in liver disease. J Hepatol 50: 604-620, 2009.

31. Rosmorduc O and Housset C: Hypoxia: A link between fibrogenesis, angiogenesis, and carcinogenesis in liver disease. Semin Liver Dis 30: 258-270, 2010.

32. Shi L, Wu LL, Yang JR, Chen XF, Zhang Y, Chen ZQ, Liu CL, Chi SY, Zheng JY, Huang HX, et al: Serum peroxiredoxin3 is a useful biomarker for early diagnosis and assessemnt of prognosis of hepatocellular carcinoma in Chinese patients. Asian Pac J Cancer Prev 15: 2979-2986, 2014.

33. Thomas MB, Jaffe D, Choti MM, Belghiti J, Curley S, Fong Y, Gores G, Kerlan R, Merle P, O'Neil B, et al: Hepatocellular carcinoma: Consensus recommendations of the national cancer institute clinical trials planning meeting. J Clin Oncol 28: 3994-4005, 2010. 